\title{
A Subclass of Meromorphic Close-to-Convex Functions of Janowski's Type
}

\author{
Young Jae Sim and Oh Sang Kwon \\ Department of Mathematics, Kyungsung University, Busan 608-736, Republic of Korea \\ Correspondence should be addressed to Oh Sang Kwon, oskwon@ks.ac.kr
}

Received 9 April 2012; Accepted 19 June 2012

Academic Editor: Aloys Krieg

Copyright (C 2012 Y. J. Sim and O. S. Kwon. This is an open access article distributed under the Creative Commons Attribution License, which permits unrestricted use, distribution, and reproduction in any medium, provided the original work is properly cited.

We introduce a subclass $\Sigma(A, B)(-1 \leq B<A \leq 1)$ of functions which are analytic in the punctured unit disk and meromorphically close-to-convex. We obtain some coefficients bounds and some argument and convolution properties belonging to this class.

\section{Introduction}

Let $\mathscr{H}(\mathbb{U})$ be the set of all analytic functions on the open unit disk $\mathbb{U}:=\{z:|z|<1\}$, and let $\mathscr{A}$ be the subclass of $\mathscr{H}(\mathbb{U})$ which contains the functions normalized by $f(0)=0$ and $f^{\prime}(0)=1$. A function $f(z) \in \mathscr{A}$ is said to be starlike of order $\alpha$ if and only if

$$
\mathfrak{R}\left\{\frac{z f^{\prime}(z)}{f(z)}\right\}>\alpha,
$$

for some $\alpha(0 \leq \alpha<1)$ and for all $z \in \mathbb{U}$. The class of starlike functions of order $\alpha$ is denoted by $\mathcal{S}^{*}(\alpha)$.

If $f$ and $g$ are analytic in $\mathbb{U}$, we say that $f$ is subordinate to $g$, written as follows:

$$
f \prec g \quad \text { in } \mathbb{U} \quad \text { or } \quad f(z) \prec g(z) \quad(z \in \mathbb{U}) \text {, }
$$

if there exists a Schwarz function $w(z)$, which is analytic in $\mathbb{U}$ with

$$
w(0)=0, \quad|w(z)|<1 \quad(z \in \mathbb{U})
$$


such that

$$
f(z)=g(w(z)) \quad(z \in \mathbb{U})
$$

In particular, if the function $g$ is univalent in $\mathbb{U}$, the above subordination is equivalent to

$$
f(0)=g(0), \quad f(\mathbb{U}) \subset g(\mathbb{U})
$$

The Hadamard product (or convolution) of two series

$$
f(z)=\sum_{n=0}^{\infty} a_{n} z^{n}, \quad g(z)=\sum_{n=0}^{\infty} b_{n} z^{n}
$$

is defined by

$$
(f * g)(z)=\sum_{n=0}^{\infty} a_{n} b_{n} z^{n}
$$

For a convex function $f$, it follows from Alexander's Theorem that $z f^{\prime}(z)=f(z) *\left(z /(1-z)^{2}\right)$ is a starlike function. In view of the identity $f(z)=f(z) *(z /(1-z))$, it is then clear that the classes of convex and starlike functions can be unified by considering functions $f$ satisfying $f * g$ is starlike for a fixed function $g \in \mathcal{A}$.

Though the convolution of two univalent (or starlike) functions does not need be univalent, it is well-known that the classes of starlike, convex, and close-to-convex functions are closed under convolution with convex functions. These results were later extended to convolution with prestarlike functions. For $\alpha<1$, the class $\mathcal{R}_{\alpha}$ of prestarlike functions of order $\alpha$ is defined by

$$
\mathcal{R}_{\alpha}:=\left\{f \in \mathcal{A}: f * \frac{z}{(1-z)^{2-2 \alpha}} \in \mathcal{S}^{*}(\alpha)\right\},
$$

while $\mathcal{R}_{1}$ consists of $f \in \mathcal{A}$ satisfying $\Re f(z) / z>1 / 2$.

By using the convex hull method $[1,2]$ and the method of differential subordination [3], Shanmugam [4] introduced and investigated convolution properties of various subclasses of analytic functions. Ali et al. [5] and Supramaniam et al. [6] investigated these properties for subclasses of multivalent starlike and convex functions. And Chandrashekar et al. [7] also investigated these properties for the functions with respect to symmetric points, conjugate, or symmetric conjugate points. More results using the convex hull method and the method of differential subordination can be found in $[8,9]$.

Let $\Sigma$ denote the class of all univalent meromorphic functions $f(z)$ normalized by

$$
f(z)=\frac{1}{z}+\sum_{k=1}^{\infty} a_{k} z^{k}
$$


which are analytic in the punctured unit disk

$$
\mathbb{U}^{*}=\{z: z \in \mathbb{C}, 0<|z|<1\}=\mathbb{U}-\{0\} .
$$

We denote by $\Sigma^{*}(\alpha)$ the subclass of $\Sigma$ consisting of $g(z)$ formed by

$$
g(z)=\frac{1}{z}+\sum_{k=1}^{\infty} b_{k} z^{k}
$$

which are meromorphic starlike of order $\alpha$ in $\mathbb{U}^{*}$. In particular, we denote by $\Sigma^{*}$, when $\alpha=0$. Also, a function $f(z)$ of the form (1.9) is said to be meromorphic close-to-convex in $\mathbb{U}^{*}$ if there is a $g(z)$ in $\Sigma^{*}$ such that

$$
\Re\left\{\frac{z f^{\prime}(z)}{g(z)}\right\}<0 \quad(z \in \mathbb{U}) .
$$

We denote $\Sigma_{k}$ the set of functions close-to-convex in $\mathbb{U}^{*}$.

In a recent paper, Gao and Zhou [10] introduced an interesting subclass $\mathcal{K}_{s}$ of the analytic function class $\mathcal{A}$ and the univalent function $\mathcal{S}$, which contains the functions $f$ satisfying the following inequality:

$$
\mathfrak{R}\left\{\frac{z^{2} f^{\prime}(z)}{g(z) g(-z)}\right\}<0 \quad(z \in \mathbb{U}),
$$

for some $g(z) \in \mathcal{S}^{*}(1 / 2)$. Here, $\mathcal{S}^{*}(1 / 2)$ is the class of starlike functions of order $1 / 2$ in $\mathbb{U}$. After that, many classes related to $\mathcal{K}_{S}$ investigated and studied by some authors. Especially, Wang et al. [11, 12], Kowalczyk and Leś-Bomba [13], Xu et al. [14], and Seker [15] introduced the generalized class of the class $\mathcal{K}_{s}$. And they gave some properties of analytic functions in each classes.

In this paper, we define a class of meromorphic functions of Janowski's type, related to the meromorphically close-to-convex functions as follows.

Definition 1.1. Let $f \in \Sigma$ and be given by (1.9) and $-1 \leq B<A \leq 1$. Then $f \in \Sigma(A, B)$, if there exists $g(z) \in \Sigma^{*}(1 / 2)$ such that for $z \in \mathbb{U}$,

$$
\frac{f^{\prime}(z)}{g(z) g(-z)} \prec \frac{1+A z}{1+B z}
$$

Theorem A. If $g(z) \in \Sigma^{*}(1 / 2)$, then $-z g(z) g(-z) \in \Sigma^{*}$.

Proof. At first, we know that

$$
-\mathfrak{R}\left\{\frac{z g^{\prime}(z)}{g(z)}\right\}>\frac{1}{2}
$$


for $z \in \mathbb{U}$, since $g \in \Sigma^{*}(1 / 2)$. And let $h(z)=-z g(z) g(-z)$. Then

$$
\frac{z h^{\prime}(z)}{h(z)}=1+\frac{z g^{\prime}(z)}{g(z)}-\frac{z g^{\prime}(-z)}{g(-z)}
$$

So we have

$$
-\mathfrak{R}\left\{\frac{z h^{\prime}(z)}{h(z)}\right\}=1-\mathfrak{R}\left\{\frac{z g^{\prime}(z)}{g(z)}\right\}-\mathfrak{R}\left\{\frac{-z g^{\prime}(-z)}{g(-z)}\right\}>-1+\frac{1}{2}+\frac{1}{2}
$$

Hence $h \in \Sigma^{*}$.

Therefore, we know that $\Sigma(A, B)$ is a subclass of $\Sigma_{k}$, by Theorem A.

We will obtain some coefficient bounds and some argument and convolution properties belonging to this class.

\section{On the Coefficient Estimates of Functions in $\Sigma(A, B)$}

Now we give the coefficient estimates of functions in $\Sigma(A, B)$. We need the following Lemma to estimate of coefficient of functions in $\Sigma(A, B)$.

Lemma 2.1. Let $g \in \Sigma^{*}(1 / 2)$ and be given by (1.11), then one has

$$
\left|2 b_{2 n-1}-2 b_{0} b_{2 n-2}+2 b_{1} b_{2 n-3}-\cdots+(-1)^{n-1} 2 b_{n-2} b_{n}+(-1)^{n} b_{n-1}^{2}\right| \leq \frac{1}{n} .
$$

Proof. According to the Theorem A, we have $-z g(z) g(-z) \in \Sigma^{*}$, and if let

$$
G(z)=-z g(z) g(-z)
$$

we know that $G(-z)=-G(z)$, so $G(z)$ is an odd meromorphic starlike function. If we let

$$
G(z)=\frac{1}{z}+\sum_{n=1}^{\infty} C_{2 n-1} z^{2 n-1}
$$

it's well-known [16, Volume 2, page 232] that

$$
\left|C_{2 n-1}\right| \leq \frac{1}{n}
$$

Substituting the series expressions of $g(z), G(z)$ in (2.2) and comparing the coefficients of two side of this equation, using (2.3) we can get an equality:

$$
C_{2 n-1}=2 b_{2 n-1}-2 b_{0} b_{2 n-2}+2 b_{1} b_{2 n-3}-\cdots+(-1)^{n-1} 2 b_{n-2} b_{n}+(-1)^{n} b_{n-1}^{2} \text {, }
$$

and the proof of Lemma 2.1 is completed. 
International Journal of Mathematics and Mathematical Sciences

Theorem 2.2. Let $f(z)$ given by (1.9) and $-1 \leq B<A \leq 1$. If

$$
\sum_{n=1}^{\infty}\left\{(1+|B|) n\left|a_{n}\right|+(1+|A|) \frac{1}{n}\right\} \leq A-B
$$

then $f \in \Sigma(A, B)$.

Proof. Let the functions $f(z)$ and $g(z)$ be given by (1.9) and (1.11), respectively. Furthermore, we let $g(z) \in \Sigma^{*}(1 / 2)$. Then

$$
G(z)=-z g(z) g(-z)=\frac{1}{z}+\sum_{n=1}^{\infty} C_{2 n-1} z^{2 n-1}
$$

where $C_{2 n-1}$ is given by (2.5) and $\left|C_{2 n-1}\right| \leq 1 / n$. Now we obtain

$$
\begin{aligned}
\Delta & :=\left|z f^{\prime}(z)-z g(z) g(-z)\right|-\left|A z f^{\prime}(z)-B z g(z) g(-z)\right| \\
& =\left|\sum_{n=1}^{\infty} n a_{n} z^{n}+\sum_{n=1}^{\infty} C_{2 n-1} z^{2 n-1}\right|-\left|(B-A) \frac{1}{z}-\sum_{n=1}^{\infty} A C_{2 n-1} z^{2 n-1}-\sum_{n=1}^{\infty} B n a_{n} z^{n}\right| .
\end{aligned}
$$

Thus, for $|z|=r(0 \leq r<1)$, we have from (2.6)

$$
\begin{aligned}
\Delta & \leq \sum_{n=1}^{\infty} n\left|a_{n}\right| r^{n}+\sum_{n=1}^{\infty}\left|C_{2 n-1}\right| r^{2 n-1}-\left((A-B) \frac{1}{r}-\sum_{n=1}^{\infty}\left|A C_{2 n-1}\right| r^{2 n-1}-\sum_{n=1}^{\infty} n\left|B a_{n}\right| r^{n}\right) \\
& =\sum_{n=1}^{\infty}(1+|B|) n\left|a_{n}\right| r^{n}+\sum_{n=1}^{\infty}(1+|A|)\left|C_{2 n-1}\right| r^{2 n-1}-(A-B) \frac{1}{r} \\
& \leq \sum_{n=1}^{\infty}(1+|B|) n\left|a_{n}\right| r^{n}+\sum_{n=1}^{\infty}(1+|A|) \frac{1}{n} r^{2 n-1}-(A-B) \frac{1}{r} \\
& \leq 0 .
\end{aligned}
$$

Thus we have

$$
\left|z f^{\prime}(z)-z g(z) g(-z)\right|<\left|A z g(z) g(-z)-B z f^{\prime}(z)\right|
$$

which is equivalent to

$$
\left|\frac{f^{\prime}(z)}{g(z) g(-z)}-1\right|<\left|A-B \frac{f^{\prime}(z)}{g(z) g(-z)}\right|
$$

which implies that $f \in \Sigma(A, B)$. 
Theorem 2.3. Let $f(z) \in \Sigma(A, B)(-1 \leq B<A \leq 1)$ and $g(z)$ is given by (1.9) and (1.11), respectively. Then for $k \geq 1$ one has

$$
\begin{aligned}
& 4 k^{2}\left|a_{2 k}\right|^{2}+\sum_{n=1}^{k-1} 4\left(1-B^{2}\right) n^{2}\left|a_{2 n}\right|^{2} \\
& \quad+\sum_{n=1}^{k}\left(\left(1-B^{2}\right)(2 n-1)^{2}\left|a_{2 n-1}\right|^{2}+2(1-A B)(2 n-1) \Re\left\{a_{2 n-1} \overline{C_{2 n-1}}\right\}+\left(1-A^{2}\right)\left|C_{2 n-1}\right|^{2}\right) \\
& \leq(A-B)^{2},
\end{aligned}
$$

where $C_{2 k-1}$ is given by (2.5).

Proof. Let $f(z) \in \Sigma(A, B)$. Then we have

$$
-\frac{z f^{\prime}(z)}{G(z)}=\frac{1+A w(z)}{1+B w(z)}
$$

where $w$ is an analytic function in $\mathbb{U},|w(z)|<1$ for $z \in \mathbb{U}$ and $G(z)=-z g(z) g(-z)$. Then,

$$
-z f^{\prime}(z)-G(z)=\left(A \cdot G(z)+B z f^{\prime}(z)\right) w(z) .
$$

Thus, putting

$$
w(z)=\sum_{n=1}^{\infty} t_{n} z^{n}
$$

we obtain

$$
\begin{aligned}
-\sum_{n=1}^{\infty} n a_{n} z^{n}-\sum_{n=1}^{\infty} C_{2 n-1} z^{2 n-1} & \\
= & \left\{(A-B) \frac{1}{z}+\sum_{n=1}^{\infty} A \cdot C_{2 n-1} z^{2 n-1}+\sum_{n=1}^{\infty} n B a_{n} z^{n}\right\}\left\{\sum_{n=1}^{\infty} t_{n} z^{n}\right\} .
\end{aligned}
$$

With comparing the coefficients, we can write

$$
\begin{aligned}
-\sum_{n=1}^{2 k} n a_{n} z^{n}-\sum_{n=1}^{k} C_{2 n-1} z^{2 n-1}+\sum_{n=2 k+1}^{\infty} c_{n} z^{n} \\
\quad=\left\{(A-B) \frac{1}{z}+\sum_{n=1}^{k} A \cdot C_{2 n-1} z^{2 n-1}+\sum_{n=1}^{2 k-1} n B a_{n} z^{n}\right\}\left\{\sum_{n=1}^{\infty} t_{n} z^{n}\right\} .
\end{aligned}
$$


Then, squaring the modulus of the both sides of the above equality and integrating along $|z|=r$ and using the fact the $|w(z)|<1$, we obtain

$$
\begin{aligned}
& \sum_{n=1}^{k}\left|(2 n-1) a_{2 n-1}+C_{2 n-1}\right|^{2} r^{4 n-2}+\sum_{n=1}^{k}\left|2 n a_{2 n}\right|^{2} r^{4 n} \\
& \quad<(A-B)^{2} \frac{1}{r^{2}}+\sum_{n=1}^{k}\left|A C_{2 n-1}+B(2 n-1) a_{2 n-1}\right|^{2} r^{4 n-2}+\sum_{n=1}^{k-1}\left|2 B n a_{2 n}\right|^{2} r^{4 n}
\end{aligned}
$$

Letting $r \rightarrow 1$ on the both sides, we obtain

$$
\begin{aligned}
& \sum_{n=1}^{k}\left|(2 n-1) a_{2 n-1}+C_{2 n-1}\right|^{2}-\sum_{n=1}^{k}\left|A C_{2 n-1}+B(2 n-1) a_{2 n-1}\right|^{2} \\
& +\sum_{n=1}^{k}\left|2 n a_{2 n}\right|^{2}-\sum_{n=1}^{k-1}\left|2 B n a_{2 n}\right|^{2} \leq(A-B)^{2}
\end{aligned}
$$

which implies the inequality (2.12).

Lemma 2.4 (see [2, Ruscheweyh and Sheil-Small]). Let $\varphi$ and $\psi$ be convex in $\mathbb{U}$ and suppose $f \prec \psi$. Then $\varphi * f<\varphi * \psi$.

Theorem 2.5. If $f \in \Sigma(A, B)(-1 \leq B<A \leq 1)$, then there exists $p<(1+A z) /(1+B z)$ such that for all $s$ and $t$ with $|s| \leq 1$ and $|t| \leq 1$,

$$
\frac{t^{2} f^{\prime}(t z) p(s z)}{s^{2} f^{\prime}(s z) p(t z)}<\left(\frac{1-t z}{1-s z}\right)^{2}
$$

Proof. By definition, for $f \in \Sigma(A, B)$, there exist $g(z)$ and $p(z)$ such that $g \in \Sigma^{*}(1 / 2)$,

$$
\begin{gathered}
p(z) \prec \frac{1+A z}{1+B z}, \\
\frac{f^{\prime}(z)}{g(z) g(-z)}=p(z) .
\end{gathered}
$$

Put $G(z)=-z g(z) g(-z)$, then

$$
-\frac{z f^{\prime}(z)}{G(z)}=p(z)
$$

And (2.22) implies that

$$
-\frac{z f^{\prime \prime}(z)}{f^{\prime}(z)}+\frac{z p^{\prime}(z)}{p(z)}-2=-\frac{z G^{\prime}(z)}{G(z)}-1
$$


Since $G(z) \in \Sigma^{*}$,

$$
-\frac{z G^{\prime}(z)}{G(z)} \prec \frac{1+z}{1-z}
$$

hence

$$
-\frac{z G^{\prime}(z)}{G(z)}-1 \prec \frac{2 z}{1-z}
$$

For $s$ and $t$ such that $|s| \leq 1,|t| \leq 1$, the function

$$
h(z)=\int_{0}^{z}\left(\frac{s}{1-s u}-\frac{t}{1-t u}\right) d u
$$

is convex in $\mathbb{U}$. Applying Lemma 2.4 with this $h$, we have

$$
\left(-\frac{z f^{\prime \prime}(z)}{f^{\prime}(z)}+\frac{z p^{\prime}(z)}{p(z)}-2\right) * h(z) \prec \frac{2 z}{1-z} * h(z)
$$

Given any function $l(z)$ analytic in $U$ with $l(0)=0$, we have

$$
(l * h)(z)=\int_{t z}^{s z} l(u) \frac{d u}{u}(z \in \mathbb{U})
$$

so (2.27) reduces to

$$
\log \left(\frac{t^{2} f^{\prime}(t z) p(s z)}{s^{2} f^{\prime}(s z) p(t z)}\right) \prec \log \left(\frac{1-t z}{1-s z}\right)^{2}
$$

Exponentiating both sides of (2.29) leads to (2.20).

\section{Argument and Convolution Properties of Functions in $\Sigma(A, B)$}

In this section, we will solve some problems related to argument and convolution properties of functions in the class $\Sigma(A, B)$. To solve these problems, we need the following Lemmas.

Lemma 3.1 (see [17, Goluzin], [3, page 62]). Let $f$ be an analytic function in $\mathbb{U}$ which is normalized and starlike, then

$$
\mathfrak{R}\left\{\frac{f(z)}{z}\right\}^{1 / 2}>\frac{1}{2}
$$

This inequality is sharp with extremal function $f(z)=z /(1-z)^{2}$. 
Lemma 3.2 (see [1, Ruscheweyh]). Let $\alpha \leq 1, \phi \in \mathcal{R}_{\alpha}$ and $f \in \mathcal{S}^{*}(\alpha)$. Then

$$
\frac{\phi * H f}{\phi * f}(\mathbb{U}) \subset \overline{\mathrm{co}}(H(\mathbb{U}))
$$

for any analytic function $H \in \mathscr{H}(\mathbb{U})$, where $\overline{\mathrm{co}}(H(\mathbb{U}))$ denote the closed convex hull of $H(\mathbb{U})$.

Theorem 3.3. Let $f(z) \in \Sigma(A, B)(-1 \leq B<A \leq 1)$. Then

$$
\left|\arg \left(-z^{2} f^{\prime}(z)\right)\right| \leq \arcsin \frac{(A-B) r}{1-A B r^{2}}+2 \arcsin r \quad(|z|=r)
$$

Proof. Let $f(z) \in \Sigma(A, B)$. Then there exists a function $g(z) \in \Sigma^{*}(1 / 2)$ satisfying

$$
\frac{f^{\prime}(z)}{g(z) g(-z)}=p(z)
$$

where

$$
p(z) \prec \frac{1+A z}{1+B z}
$$

And $p(|z|<r)$ is contained in the disk

$$
\left|w-\frac{1-A B r^{2}}{1-B^{2} r^{2}}\right|<\frac{(A-B) r}{1-B^{2} r^{2}}
$$

from which it follows that

$$
|\arg p(z)| \leq \arcsin \frac{(A-B) r}{1-A B r^{2}}
$$

Hence we can obtain the inequality:

$$
\left|\arg \left(-z^{2} f^{\prime}(z)\right)\right| \leq \arcsin \frac{(A-B) r}{1-A B r^{2}}+|\arg (z g(z))|+|\arg (-z g(-z))|
$$

Hence it suffices to find the upper bounds of $|\arg (z g(z))|$. Since $g \in \Sigma^{*}(1 / 2)$, we have $g(z) \neq 0$ for $z \in \mathbb{U}^{*}$ and $h \equiv 1 / g \in \mathcal{S}^{*}(1 / 2)$. If we define $k(z)=(g(z))^{2} / z$, then $k \in \mathcal{S}^{*}$ and we can apply Lemma 3.1 to obtain

$$
\mathfrak{R}\left\{\frac{k(z)}{z}\right\}^{1 / 2}>\frac{1}{2} .
$$


From the relation between $g, h$ and $k$ we obtain

$$
z g(z) \prec 1+z \quad(z \in \mathbb{U})
$$

which implies

$$
|z g(z)-1| \leq r \quad(|z|=r)
$$

Hence

$$
|\arg (z g(z))| \leq \arcsin r \quad(|z|=r)
$$

for $z(|z|=r)$, and so the result is proved.

Theorem 3.4. Let $f \in \Sigma(A, B)(-1 \leq B<A \leq 1)$ satisfying the condition

$$
\frac{1}{2}<-\Re\left\{\frac{z g^{\prime}(z)}{g(z)}\right\}<\frac{3}{2}-\frac{1}{2} \alpha
$$

and $\phi \in \Sigma$ with $z^{2} \phi(z) \in \mathcal{R}_{\alpha}$. Then $\phi * f \in \Sigma(A, B)$.

Proof. Let $f \in \Sigma(A, B)$ such that

$$
\frac{f^{\prime}(z)}{g(z) g(-z)} \prec \frac{1+A z}{1+B z}
$$

and define the functions $G$ and $H$ by

$$
G(z):=-z g(z) g(-z), \quad H(z):=-\frac{z f^{\prime}(z)}{G(z)} .
$$

Thus for any fixed $z \in \mathbb{U}$,

$$
H(z) \prec \frac{1+A z}{1+B z} .
$$

From the condition (3.13), we can easily obtain that

$$
-\mathfrak{R}\left\{\frac{z f^{\prime}(z)}{G(z)}\right\}<2-\alpha,
$$


and this inequality implies that $z^{2} G(z) \in \mathcal{S}^{*}(\alpha)$. Put $\varphi(z)=(\phi * G)(z)$. Since $G(z)$ is an odd meromorphic starlike function, so $\varphi(z)$ is. Define a function $k$ by $k(z)=\sqrt{z \varphi(z)} / z$. Since $\varphi$ is an odd meromorphic starlike function, $k \in \Sigma^{*}(1 / 2)$ and $\varphi(z)=-z k(z) k(-z)$. Hence

$$
\begin{aligned}
\frac{(\phi * f)^{\prime}(z)}{k(z) k(-z)} & =\frac{-z(\phi * f)^{\prime}(z)}{\varphi(z)} \\
& =\frac{\phi(z) *\left(-z f^{\prime}(z)\right)}{(\phi * G)(z)} \\
& =\frac{\phi(z) * H(z) G(z)}{(\phi * G)(z)} \\
& =\frac{z^{2} \phi(z) * H(z) z^{2} G(z)}{z^{2} \phi(z) * z^{2} G(z)} .
\end{aligned}
$$

Since $z^{2} \phi(z) \in \mathcal{R}_{\alpha}$ and $z^{2} G(z) \in \mathcal{S}^{*}(\alpha)$, Lemma 3.2 yields

$$
\frac{z^{2} \phi(z) * H(z) z^{2} G(z)}{z^{2} \phi(z) * z^{2} G(z)} \in \overline{\mathrm{co}}(H(\mathbb{U}))
$$

where $\overline{\mathrm{co}}(H(\mathbb{U}))$ denote the closed convex hull of $H(\mathbb{U})$, and because

$$
H(z) \prec \frac{1+A z}{1+B z} \quad(z \in \mathbb{U})
$$

and $(1+A z) /(1+B z)$ is convex in $\mathbb{U}$, it follows that

$$
\frac{(\phi * f)^{\prime}(z)}{k(z) k(-z)} \prec \frac{1+A z}{1+B z}
$$

in $\mathbb{U}$. Thus $\phi * f \in \Sigma(A, B)$ and the proof of Theorem 3.4 is completed.

\section{References}

[1] S. Ruscheweyh, Convolutions in Geometric Function Theory, vol. 83 of Séminaire de Mathématiques Supérieures, Presses de l'Université de Montréal, Montreal, Canada, 1982.

[2] S. Ruscheweyh and T. Sheil-Small, "Hadamard products of Schlicht functions and the PólyaSchoenberg conjecture," Commentarii Mathematici Helvetici, vol. 48, pp. 119-135, 1973.

[3] S. S. Miller and P. T. Mocanu, Differential Subordinations. Theory and Applications, vol. 225, Marcel Dekker, New York, NY, USA, 2000.

[4] T. N. Shanmugam, "Convolution and differential subordination," International Journal of Mathematics and Mathematical Sciences, vol. 12, no. 2, pp. 333-340, 1989.

[5] R. M. Ali, V. Ravichandran, and S. K. Lee, "Subclasses of multivalent starlike and convex functions," Bulletin of the Belgian Mathematical Society, vol. 16, no. 3, pp. 385-394, 2009.

[6] S. Supramaniam, R. M. Ali, S. K. Lee, and V. Ravichandran, "Convolution and differential subordination for multivalent functions," Bulletin of the Malaysian Mathematical Sciences Society, vol. 32, no. 3, pp. 351-360, 2009. 
[7] R. Chandrashekar, R. M. Ali, S. K. Lee, and V. Ravichandran, "Convolutions of meromorphic multivalent functions with respect to n-ply points and symmetric conjugate points," Applied Mathematics and Computation, vol. 218, no. 3, pp. 723-728, 2011.

[8] K. S. Padmanabhan and R. Manjini, "Certain applications of differential subordination," Publications de l'Institut Mathématique, vol. 39, no. 53, pp. 107-118, 1986.

[9] K. S. Padmanabhan and R. Parvatham, "Some applications of differential subordination," Bulletin of the Australian Mathematical Society, vol. 32, no. 3, pp. 321-330, 1985.

[10] C. Gao and S. Zhou, "On a class of analytic functions related to the starlike functions," Kyungpook Mathematical Journal, vol. 45, no. 1, pp. 123-130, 2005.

[11] Z.-G. Wang and D.-Z. Chen, "On a subclass of close-to-convex functions," Hacettepe Journal of Mathematics and Statistics, vol. 38, no. 2, pp. 95-101, 2009.

[12] Z.-G. Wang, C.-Y. Gao, and S.-M. Yuan, "On certain new subclass of close-to-convex functions," Matematichki Vesnik, vol. 58, no. 3-4, pp. 119-124, 2006.

[13] J. Kowalczyk and E. Les-Bomba, "On a subclass of close-to-convex functions," Applied Mathematics Letters, vol. 23, no. 10, pp. 1147-1151, 2010.

[14] Q.-H. Xu, H. M. Srivastava, and Z. Li, "A certain subclass of analytic and close-to-convex functions," Applied Mathematics Letters, vol. 24, no. 3, pp. 396-401, 2011.

[15] B. Seker, "On certain new subclass of close-to-convex functions," Applied Mathematics and Computation, vol. 218, no. 3, pp. 1041-1045, 2011.

[16] A. W. Goodman, Univalent Ffunctions. Vol. I, Mariner Publishing Co., Tampa, Fla, 1983.

[17] G. M. Goluzin, "Some estimates for coefficients of univalent functions," Matematicheskii Sbornik, vol. 3, no. 45, pp. 321-330, 1938 (Russian). 


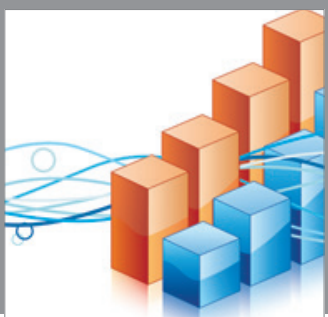

Advances in

Operations Research

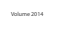

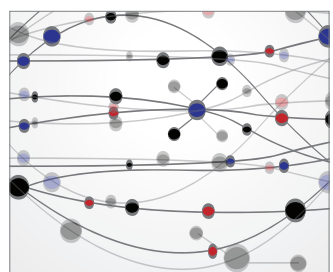

\section{The Scientific} World Journal
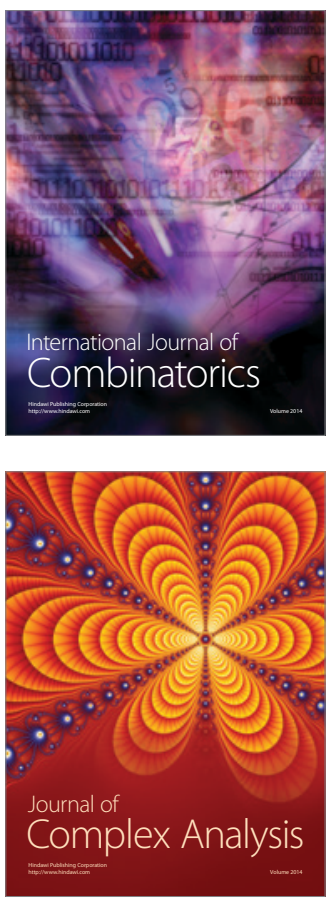

International Journal of

Mathematics and

Mathematical

Sciences
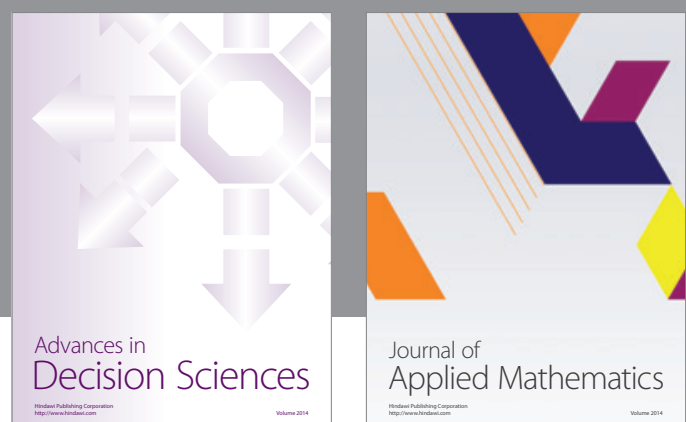

Journal of

Applied Mathematics
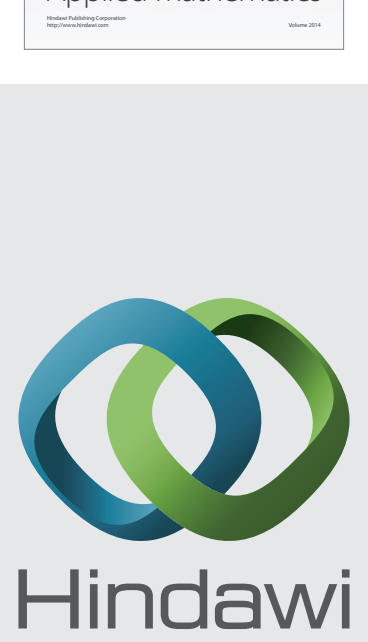

Submit your manuscripts at http://www.hindawi.com
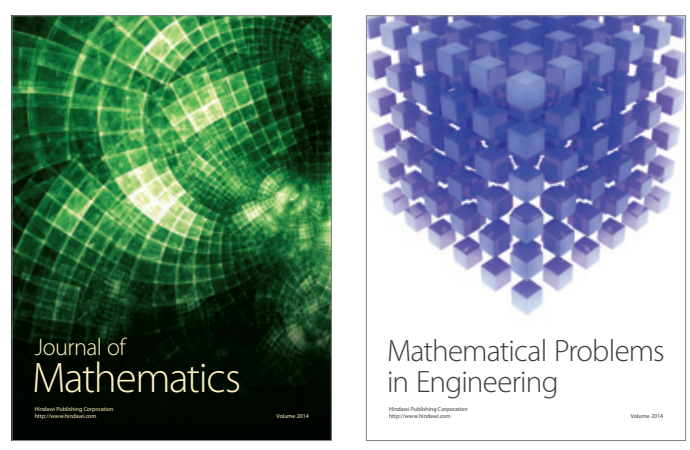

Mathematical Problems in Engineering
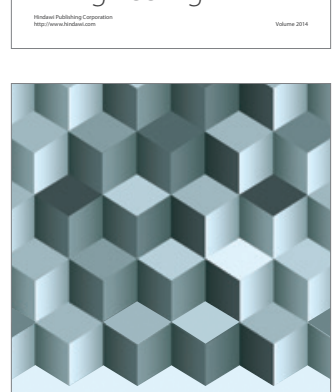

Journal of

Function Spaces
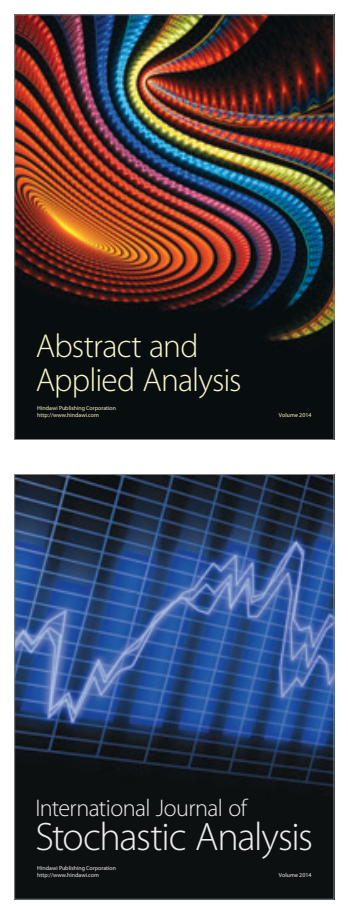

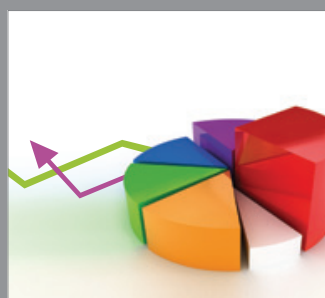

ournal of

Probability and Statistics

Promensencen
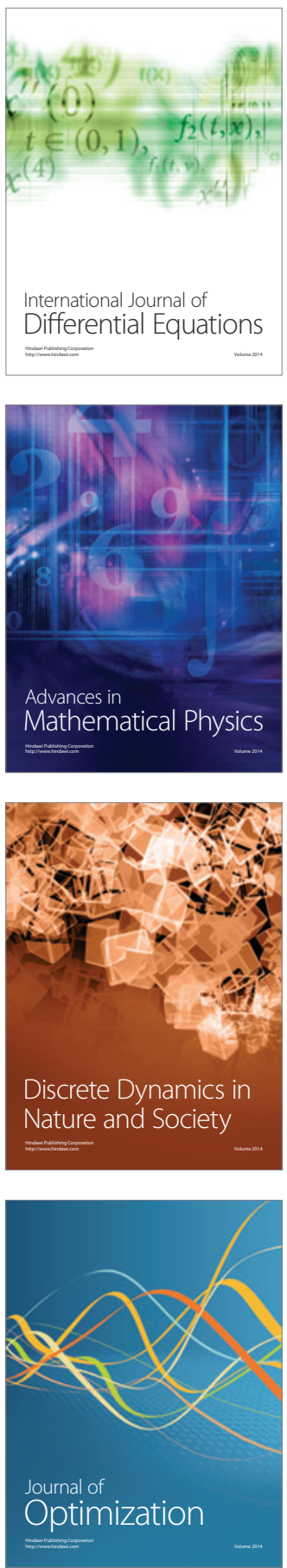